\title{
WIE EVALUIERT MAN ORGANISATORISCHEN WANDEL IN EINEM KOMPLEXEN UMFELD?
}

\author{
ZENTRALE ERGEBNISSE AUS DER EVALUIERUNG VON \\ FFG (FORSCHUNGSFÖRDERUNGSGESELLSCHAFT) UND \\ AWS (AUSTRIAN WIRTSCHAFTSSERVICE)
}

SUSANNE BÜHRER, PETER KAUFMANN, STEPHANIE DAIMER, SASCHA RUHLAND, KNUT KOSCHATZKY UND SONJA SHEIKH

\section{ABSTRACT}

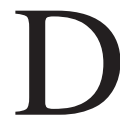
ie Strukturreform der Förderorganisationen der Jahre 2002 2004 mündete in eine Zusammenfassung von acht Vorgängerorganisationen in die neu gegründeten Agenturen aws und FFG. Die Evaluierung dazu ergab, dass die in den beiden Agenturen etablierten Strukturen und Prozesse zu einer Reduktion der organisatorischen Komplexität sowie einer Reduzierung von Koordinationsproblemen führten. Die inhaltliche Komplexität hat sich jedoch über die Jahre aufgrund des förderpolitischen Umfeldes tendenziell eher erhöht; dies hat jedoch wenig mit den Strukturreformen und der Entwicklung der Agenturen zu tun. Die politische Steuerungsfähigkeit gegenüber den Zielgruppen ist nun zwar höher, bleibt aber unter ihrem Potenzial, da die Agenturen derzeit noch über zu wenig Freiraum zur Weiterentwicklung ihres Portfolios verfügen. Die Agenturen weisen auch einen hohen Professionalisierungsgrad auf, der mittelbar auch eine Verbesserung der Umsetzungschancen von Regierungszielen bedeutet. Eine Weiterentwicklung gemäß dem Leitsatz "Leistungsfähigkeit durch Eigenverantwortung" hinsichtlich der Stärkung der finanziellen und operationalen Autonomie der Agenturen ist jedoch anzuraten, wenn die Agenturen die Entwicklung des österreichischen Innovationssystems hin zu einem Innovation Leader so effektiv wie möglich unterstützen sollen.

Keywords: institutionelle Evaluation, Förderagenturen

\section{ANLASS UND ZIELSETZUNG DER EVALUIERUNG}

Im Juni 2016 wurden das Fraunhofer ISI und die KMU Forschung Austria von den beiden damaligen Eigentümer-Ressorts bmvit (Bundesministerium für Verkehr, Innovation und Technologie) und bmwfw (Bundesministerium für Wirtschaft, Forschung und Wissenschaft) mit der Evaluierung der Förderungsgesellschaften FFG und aws beauftragt. Ziel war es, über zehn Jahre nach Umsetzung der Strukturreformen Bilanz über deren Zielerreichung zu ziehen. Die Evaluierung wurde im Juni 2017 abgeschlossen.

Die Ziele der in den Jahren 2002 bis 2004 umgesetzten Strukturreform der österreichischen Förderagenturen im Bereich Forschung-, Technologie und Innovation bestanden in der (a) Reduktion organisatorischer und inhaltlicher Komplexität, (b) Lösung immanenter Koordinationsprobleme durch die Schaffung von ressortübergreifenden Förderungsgesellschaften, (c) Erhöhung der politischen Steuerungsfähigkeit gegenüber den Zielgruppen, (d) Verbesserung der Umsetzungschancen von Regierungszielen, nämlich Effizienz und Intensivierung des Ressourceneinsatzes. Diese vier Ziele werden für die empirische Umsetzung der Evaluierung in vier Leitthemen zusammengefasst und untersucht, (1) wie sich die interne Struktur und Organisation der Agenturen darstellt, (2) welche Unternehmenskulturen sich in den Agenturen herausgebildet haben, (3) wie sich die Agenturen innerhalb des österreichischen Innovationssystems positionieren und (4) wie die Agenturen von den Eigentümerressorts gesteuert werden. Zusätzlich hat das Evaluationsteam drei Zukunftsoptionen für die Ausgestaltung der Beziehungen zwischen Eigentümerressorts und Agenturen entwickelt.

\section{EVALUATIONSANSATZ UND METHODISCHES VORGEHEN}

Die Evaluierung erforderte eine Vorgehensweise, die der Komplexität des Untersuchungsgegenstandes gerecht wird: Organisatorischer Wandel unterliegt einer Vielzahl von Einflussfaktoren, es lässt sich keine kontrafaktische Situation erstellen und Organisationen folgen in ihrem Tun oftmals impliziten Werten und Normen. Die Evaluierung orientierte sich daher an der Vorgehensweise einer "Theory Based Evaluation" (Weiss 1997a, 1997b, Leeuw 2003, 2012), d.h. sie konzentrierte sich auf die Untersuchung der Fragen, welche Faktoren zu einer beobachteten 
Veränderung beitragen, warum und wie? Die konzeptionelle Basis für die Analyse der Umsetzung der Strukturreformziele wurde unterschiedlichen Ansätzen entnommen, die im Rahmen von vergangenen institutionellen Evaluierungen erprobt wurden. Im Einzelnen waren dies die PrincipalAgent-Theorie (Braun 1993, 1998, Braun/Gilardi 2002, Lane 2013), der Neo-Institutionalismus (z.B. Hasse/Krücken 2005, Koch 2009, Meier 2009, Meyer/Rowan 1977, Schaefers 2009) und der Unternehmenskulturansatz nach Schein (Schein 1985, 2003, Tischer/Meyer/Mayrhofer 2008, Hochfeld/Kaiser/Schraudner 2014).

Die Evaluation basierte auf einem Methodenmix bestehend aus qualitativen und quantitativen Vorgehensweisen wie Literaturanalysen, Auswertungen von Sekundärdaten, Mitarbeiter- und Zielgruppenbefragungen, narrativen ${ }^{1}$ und Leitfaden gestützten Interviews, insgesamt fünf Validierungsworkshops und einem internationalen Vergleich, der auf Dokumentenanalysen und Telefoninterviews gründete. Insgesamt wurden 73 Einzelinterviews und zwei Gruppeninterviews mit jeweils 4 Teilnehmerlnnen durchgeführt (mit Vertreterlnnen der Agenturen, der Eigentümerressorts, ExpertInnen der österreichischen FTI-Politik sowie im Rahmen eines internationalen Vergleichs) und fünf Online-Befragungen implementiert (als Mitarbeiterlnnenbefragung innerhalb der Agenturen und Eigentümerressorts sowie mit den jeweiligen Zielgruppen der Agenturen). Der Rücklauf variierte zwischen 30\% und 63\%. Mit Ausnahme der Ministerienbefragung ( $\mathrm{N}=51$ ) standen zwischen 154 und 260 Antworten für die Auswertungen zur Verfügung.

\section{DIE AGENTUREN}

Jeder der Themenschwerpunkte dieser Evaluierung wurde mit einer eigenen Zielsetzung, Methodik bzw. eigenen Leitfragen adressiert. So war es Ziel der Agenturanalyse, eine Beschreibung der internen Struktur und Organisation zu erstellen, d.h. mit Blick auf deren Aufbau- und Ablauforganisation, jedoch auch unter dem Blickwinkel von Formal- und Arbeitsstruktur. Die Analyse der Unternehmenskultur der FFG bzw. der aws fokussierte auf die zugrundeliegenden Werte und Normen in den jeweiligen Förderorganisationen. Bei der Untersuchung der ,politischen Steuerungsfähigkeit gegenüber den Zielgruppen' bzw. der Zielgruppenerreichung stand die Veränderung der Zielgruppenansprache und -koordination inkl. der Wahrnehmung der Strukturreformen durch die (potentiellen) Förderungsnehmerlnnen von FFG und aws im Vordergrund. Die Steuerung durch die Eigentümerressorts wurde vor allem im Hinblick auf die jeweiligen Selbst- und Fremdbilder von Agenturen und Ressorts sowie die formalen Rahmenbedingungen hin betrachtet.

Die wichtigsten Ergebnisse der Agenturanalyse lassen sich wie folgt zusammenfassen: Beide Agenturen agieren nach einem erfolgreich vollzogenen Fusionsprozess hochprofessionell und effizient. Im Zuge der Fusion und der dadurch entstehenden relativ großen Organisationseinheiten wurden zur Gewährleistung einer hohen Professionalität, Serviceorientierung, Effektivität und Effizienz zahlreiche Prozesse und Strukturen entwickelt und implementiert, die sich im Großen und Ganzen bewährt haben. Beide Agenturen haben erfolgreich Qualitätskon- trollen aufgebaut, Systeme zur Mitarbeiterqualifizierung etabliert und eine hohe Praxisnähe aufgebaut. Die entwickelten Werkzeuge erhöhen die Effizienz des Agenturhandelns, aber sie schaffen gleichzeitig auch Inflexibilität.

Beide Agenturen, und vor allem ihre Untereinheiten bzw. Bereiche, sind durch komplexe Aufgabenstellungen geprägt, so dass sie zwar einerseits eine hohe gemeinsame Identität aufweisen, andererseits aber im Alltagshandeln durchaus unterschiedliche Orientierungen zu finden sind, die auf die große Vielfalt unterschiedlicher Beauftragungssysteme zurückzuführen sind. In Teilen spielen aber auch noch die Sozialisierungen in einer der Vorgängerorganisationen eine Rolle.

Eine Herausforderung für beide Agenturen ist eine teils kritische Einschätzung ihrer Kundlnnen zu mehreren Qualitätsmerkmalen: Zwar wird der Erfolg des Fusionsprozesses einhellig positiv gewürdigt, vor allem hinsichtlich allgemeiner Hilfestellung und Kommunikation, Unterstützung der jeweiligen Anliegen und der Klarheit von Zuständigkeiten. Bemängelt wird von den Kundlnnen hingegen Transparenz, Geschwindigkeit und Flexibilität der Entscheidungen. Diese Herausforderung anzugehen ist Aufgabe aller Beteiligten, d.h. sie wird ohne eine aktive und verbesserte Rahmensetzung durch die Eigentümerressorts nicht zu bewerkstelligen sein.

\section{POSITIONIERUNG UND KOORDINATION}

Die beiden Agenturen sind mit ihren spezifischen Geschäftsmodellen im nationalen innovationspolitischen Umfeld klar positioniert. Sie fungieren in weiten Teilen als one-stop-shop für Forschung, Technologieentwicklung und Innovation (FFG) sowie investive Unternehmensförderung und Innovationsunterstützung (aws). Eine stärkere horizontale Koordination zwischen den Agenturen im österreichischen Fördersystem wurde durch die Strukturreformen 2002-2004 erreicht. Dies trifft jedenfalls auf die nun in Form von Bereichen weitergeführten vormaligen Agenturen zu, die aufgrund der Zusammenführung vielfältige Vorteile ausschöpfen konnten.

Es gibt jedoch noch Optimierungspotenzial. Zum Beispiel können agenturinterne Prozesse nicht gänzlich standardisiert werden, wenn die Auftraggeber unterschiedliche Bedürfnisse für Dokumentation und Reporting aufweisen (z.B. Bereich Energie/Klimafonds), oder unterschiedliche Governance-Strukturen der Eigentümer die Möglichkeiten zur Harmonisierung von Organisationsstrukturen hintanhalten (z.B. EIP/ TP in der FFG). Dieses Optimierungspotenzial kann nur unter veränderten Rahmenbedingungen ausgeschöpft werden, also indem die Agenturen ihre Förderinstrumente stärker selbst definieren können und es so zu einer strategischen Themensteuerung (durch die Auftraggeber) kommen kann.

Die Zusammenarbeit und Koordination zwischen den beiden Agenturen kann, auch wenn die Schnittmenge quantitativ relativ klein ist, insofern noch geschärft werden, indem die Kriterien zur Beauftragung der

1 Im vorliegenden Fall wurden narrative Interviews in erster Linie dazu eingesetzt, eine kohärente Chronologie der Entstehung und Entwicklung der Forschungsförderungsagenturen zu entwickeln, wobei individuelle Perspektiven und Sichtweisen intakt blieben. Die entsprechend identifizierten zentralen Ereignisse und Personen dienten dann als Basis für die Entwicklung von Interviewleitfäden für entsprechend problemzentrierte Interviews, die eine Vertiefung und Objektivierung der erhobenen Informationen ermöglichten. 
einzelnen Agenturen noch expliziter gemacht werden. Damit wird man Überlappungsbereiche klein halten, aber nicht völlig beseitigen können. Da eine trennscharfe Abgrenzung nicht gänzlich möglich ist, muss eine gute Zusammenarbeit und Abstimmung an diesen Randbereichen erfolgen, die bereits im Stadium des Programmdesigns beginnt, indem die Auftraggeber mit den Agenturen eine gemeinsame Lösung suchen.

\section{STEUERUNG UND GOVERNANCE}

Ein zentraler Befund der Evaluierung hinsichtlich der Steuerung lautet, dass das gesamte Governancesystem von (anhaltend) hoher Komplexität geprägt ist. Dies ist einerseits darin begründet, dass die Steuerung der Agenturen nach wie vor über zahlreiche gleichzeitig zu bedienende Ausführungsverträge erfolgt und andererseits im Fehlen eines einheitlichen, koordinierten Steuerungsverständnisses seitens der Eigentümer. Auch daher lassen sich abweichende Selbst- und Fremdbilder bezüglich der Arbeitsteilung zwischen Ressorts und Agenturen feststellen, die insbesondere auf die unvollständige Delegation von Umsetzungsaufgaben an die Agenturen verweisen. Die verschiedenen Akteure nutzen unterschiedliche Steuerungsmechanismen und -kanäle und stimmen sich in der Regel nicht ausreichend ab, was teilweise sogar widersprüchliche Steuerungsimpulse für die Agenturen bedeutet. Die Situation wird insbesondere durch die steigende Bedeutung der Kabinette und dem direkten Zugang der Agenturen hierhin zunehmend komplexer. Damit entsteht eine Situation, in der nicht länger eindeutig klar ist, wer letztlich die Rolle des Prinzipals einnimmt und dementsprechend die Vorgaben für die Agenten/Agenturen macht. Anstatt also Unsicherheiten für alle Beteiligten zu minimieren, entstehen eher zusätzliche. Insgesamt entsteht der Eindruck, dass eine Steuerung der Agenturen als Ganzes auch aufgrund der in den Rahmenbedingungen angelegten relativen Steuerungsschwäche der Ressorts derzeit kaum möglich ist. Es scheint ein Steuerungsvakuum zu geben, das am ehesten noch die Agenturen für sich zu nutzen wissen. Auch der vielfach geäußerte Vorwurf des Mikromanagements durch die Ressorts erklärt sich nicht zuletzt als Reaktion auf die als zu schwach wahrgenommene (eigene) Steuerungskapazität.

Während die Agenturen auch bei weitgehend unveränderten Rahmenbedingungen aufgrund etwa der Kumulation strategischer Intelligenz und des Zuwachses an Einfluss stärker bzw. autonomer geworden sind, hat es weder eine adäquate Neujustierung des Systems (auch nicht infolge der Systemevaluierung) gegeben, noch eine analoge Ko-Evolution innerhalb der Ressorts. Letzteres betrifft vor allem die ausgebliebene Entwicklung eines neuen Steuerungsverständnisses und entsprechender Einstellungen sowie Fähigkeiten vor dem Hintergrund zunehmend professionalisierter Umsetzungsprozessen in den Agenturen.

\section{ZUKUNFTSOPTIONEN}

Angesichts einer hohen Änderungsdynamik in modernen Innovationssystemen müssen die Agenturen in die Lage versetzt werden, dynamisch, risikobereit und verantwortlich Förderportfolios gestalten zu können, die politisch gesetzte Prioritäten adressieren und umsetzen. Die entsprechenden Rahmenbedingungen müssen gleichzeitig die Steuerungsfähigkeit der Eigentümerressorts stärken (nicht zuletzt, um der Problematik ansonsten fehlender mittelbarer demokratischer Kontrolle und
Legitimierung zu begegnen und die Agenturen als Instrument der Umsetzung politischer Willensbildung zu erhalten) und das Governancesystem auf eine vertrauensbasierte, ergebnisorientierte Grundlage stellen.

Als Fazit legen die Evaluatorlnnen den Eigentümern daher nahe, gemäß dem Leitsatz „Leistungsfähigkeit durch Eigenverantwortung“ vor allem die finanzielle und operationale Autonomie der Agenturen zu stärken. Die Evaluierung gibt anhand von drei Zukunftsoptionen Anregungen, wie ein solcher Prozess gestaltet werden könnte und wie sich ein verändertes Verhältnis zwischen Agenturen und Eigentümern auf die vier als relevant herausgearbeiteten Dimensionen (1) Governance, (2) Finanzierung, (3) Aufgaben, und (4) Organisationsstrukturen und Managementprozesse auswirken können. Hierzu haben wir drei denkbare Ausprägungen von Agencification differenziert:

- Agencification Stufe 1: Weiterführung des Status quo,

- Agencification Stufe 2: Weiterentwicklung des Status quo,

- Agencification Stufe 3: Höhere Autonomie durch Globalsteuerung.

Die Fortführung des Status quo führt zu keinen Veränderungen und führt dementsprechend alle Stärken und Schwächen des derzeitigen Systems weiter. Obgleich die Weiterschreibung der aktuellen Situation eine Reihe von Nachteilen beinhaltet, nämlich die konsequente Perpetuierung der im vorliegenden Bericht herausgearbeiteten Probleme, gibt es auch einen Vorteil: alle Akteure haben für das geltende System entsprechende Routinen entwickelt.

Zukunftsoption 2 "Weiterentwicklung des Status quo" fokussiert auf eine Korrektur, insbesondere der auf die Governance bezogenen Aspekte, in Richtung Stärkung der Ressorts durch eine konsequentere strategische Steuerung der Agenturen, sowie gleichzeitige Stärkung der Agenturen durch eine größere Unabhängigkeit auf der operativen Ebene. Die Agenturaufgaben wären mit Ausnahme der Aufwertung des Instrumentendesigns und größeren Freiheitsgraden bei der Programmentwicklung damit weitgehend wie bisher gestaltet; der Prinzipal würde seine Kontrollaufgaben jedoch stärker wahrnehmen.

Zukunftsoption 3 manifestiert den Übergang von der Detailsteuerung hin zu einer ergebnisorientierten Steuerung „auf Abstand“, die mittels eines Globalbudgets zwar die gewünschten Wirkungen der Agentur mittels Zielformulierung und der Definition von Wirkungsindikatoren vorgibt, die Verteilung des Budgets auf Förderinstrumente jedoch großteils der Agentur überlässt. Damit erfolgt eine Übertragung von mehr Entscheidungskompetenz und Verantwortung an die Agentur, während sich der Prinzipal aufgrund der entstehenden Freiräume verstärkt auf die politisch-strategischen Aspekte, die Gestaltung von strukturierten Lernprozessen zur Minimierung von Informationsasymmetrien (durch Transparenz und Partizipation zur Unterstützung des Agenda-Settings), sowie die Controllingfunktion fokussiert.

\section{HANDLUNGSEMPFEHLUNGEN}

Den folgenden Handlungsempfehlungen muss vorangestellt werden, dass die oben zusammengefassten kritischen Befunde zwar den entsprechenden Handlungsraum abstecken, das System in vielen Teilen jedoch durchwegs gut funktioniert. Es gibt einige Evidenz dafür, dass in bestimmten Bereichen die Rahmenbedingungen aufgrund ihrer indivi- 
duellen Ausgestaltung und Interpretation eine bessere Governance und optimierte Leistungsfähigkeit ermöglichen. Dort finden sich dann auch eine eher vertrauensbasierte Arbeitsteilung sowie die oft zitierte Konzentration beider Seiten (Ressorts und Agenturen) auf ihre jeweiligen Kernaufgaben. Dennoch lassen sich aus den Befunden folgende Optimierungspotenziale ableiten:

- Die vielen ressourcenbindenden Ausführungsverträge solIten schnellstmöglich reduziert werden, um es den Agenturen und Ressorts zu ermöglichen, sich vermehrt auf ihre Kernaufgaben zu fokussieren. Dabei sollten die bestehenden Gestaltungsmöglichkeiten hinsichtlich längerer finanzieller Planungshorizonte aktiv genutzt werden. Insbesondere die entstehenden Verzögerungen bei Ausschreibungen sowie in der Bereitstellung bewilligter Mittel stellen dabei ein prioritäres Handlungsfeld dar.

- In diesem Zusammenhang ist auch die teilweise unverhältnismäßig komplexe Situation der Mittelherkunft (Ressorts, Nationalstiftung, Österreichfonds, Garantien bei aws, etc.) anzusprechen. Diese erhöht die Zahl und Komplexität der Programme und Prozesse sowie ihre Kleinteiligkeit und Planungsunsicherheit. Die Finanzierung von Programmen sollte im Sinne eines strategischen Themenmanagements (Effektivität) sowie der Optimierung der Verwaltungseffizienz einheitlich gesteuert werden. Bestenfalls durch ein Globalbudget für die Agenturen und thematische Vorgaben wie in der oben skizzierten Option 3 beschrieben.

- Darüber hinaus sollten die Eigentümer in einen begleiteten Diskurs zur Erarbeitung eines einheitlichen oder zumindest abgestimmten Steuerungsverständnisses eintreten. Darauf aufbauend wäre die Delegation der Umsetzungsaufgaben an die Agenturen und ihre damit verbundene operationale Autonomie zu klären, um eine entsprechende Standardisierung der tatsächlich gelebten Arbeitsteilung zwischen Auftraggebern und Agenturen zu ermöglichen.

- Im Sinne einer weiteren Reduktion der bestehenden (und zukünftigen) Überschneidungen zwischen den Portfolios beider Agenturen empfehlen wir, explizite Kriterien für die Vergabe des Programm-Managements an eine der beiden Agenturen zu entwickeln. Damit ließen sich die Überlappungsbereiche noch weiter reduzieren, jedoch nicht völlig beseitigen. Auch unter der Perspektive einer größeren operativen Unabhängigkeit der Agenturen kann eine Kooperation der Agenturen erwirkt werden, wenn klar kommuniziert wird, welchen Kriterien ein vorgeschlagener Instrumentenmix zu entsprechen hat.

- Die Ressorts sollten im Rahmen ihrer Eigentümerfunktion eine größere Sichtbarkeit gegenüber den Fördernehmern erwirken, etwa über entsprechende Kommunikationsregelungen im Rahmenvertrag zwischen Agentur und den Ressorts.

- Nicht zuletzt zur Stärkung der Wirkungsorientierung und der Möglichkeit des entsprechenden Nachweises, sowie der damit steigenden strategischen Intelligenz in den Ressorts wird empfohlen, entsprechende Sekundärdatenbasen in Österreich zusammenzuführen und Evaluatorlnnen zugänglich zu machen. Die Ressorts könnten diese Basis dann nutzen, um noch bessere Evidenz zur Entscheidungsunterstützung über die Weiterführung von Portfolios etc. erarbeiten und steuernd einsetzen zu können. Insbesondere eine Zusammenführung von Förderdaten der Statistik Austria, des BMF sowie der Agenturen und der Bundesländer wäre von hohem Wert.

\section{FAZIT}

Abgesehen von dem grundsätzlich positiven Befund für die Entwicklung der beiden Agenturen an sich, gibt es nach wie vor Herausforderungen, die aus Sicht des Evaluatorenteams unter anderem adressiert werden können, wenn am Verhältnis zwischen Eigentümern und Agenturen einige zentrale Änderungen vorgenommen werden.

Die Fusion der Vorläuferorganisationen als Gesamtprozess hat zu einer weitgehenden Homogenisierung innerhalb der neu geschaffenen Agenturen insbesondere mit Bezug zu den dort jeweils entwickelten und angewandten Prozessen geführt. Allerdings existieren nach wie vor deutliche Unterschiede hinsichtlich der jeweils geltenden Rahmenbedingungen wie etwa die unterschiedliche Finanzierungsbasis der einzelnen Bereiche, die Aufgabenprofile (auch und insbesondere die Arbeitsteilung mit den Eigentümern bzw. Auftraggebern), sowie die unterschiedlichen Beauftragungsverhältnisse durch die Auftraggeber.

Eine Reduktion der inhaltlichen Komplexität wurde mit der Umsetzung der Strukturreform und auch in weiterer Folge nicht erreicht. Dies ist einerseits durch die zunehmende inhaltliche Komplexität der Rahmenbedingungen (Regelungen der Europäischen Kommission, wirkungsorientierte Haushaltsführung, generelle Ausdifferenzierung der Wirtschafts- und Innovationspolitik und Erweiterung um neue Technologie- und Handlungsfelder etc.) begründet, die nicht nur für zusätzlichen Aufwand bei den Ressorts sowie Agenturen sorgte, sondern auch bei den Kundlnnen im Zuge der Beantragung und Abrechnung von Projekten. Darüber hinaus hat sich die Anzahl an beauftragten Initiativen beständig erhöht, d.h. der bereits in der Systemevaluierung beschriebene „Förderdschungel" bzw. für die Förderprofis eher der "Fördersupermarkt" (Mayer et al. 2009) existiert nach wie vor. Auch aufgrund einer stärker an Sichtbarkeit interessierten Politik, die vor allem durch zusätzliche Förderangebote hergestellt werden soll, besteht somit eine große Anzahl an Programmen, wovon manche unterkritische Größen aufwei$\operatorname{sen}^{2}$ (vgl. RH-Bericht zur Forschungsfinanzierung 2016) oder aufgrund der Interventionslogik ein Zusammenlegen mit anderen Programmen überlegenswert scheint.

Die Lösung immanenter Koordinationsprobleme durch die Schaffung von ressortübergreifenden Förderungsgesellschaften spricht mehrere Ebenen der Koordination an: (1) zwischen den Ministerien und den Agenturen im Rahmen ihrer Eigentümer- und Auftraggeberfunktion, (2) zwischen den Agenturen und den Bundesländern, und (3) zwischen den Agenturen und den Ministerien hinsichtlich der Unterstützung der Agenden der Ministerien mit der Europäischen Kommission und anderen internationalen Initiativen. Während die Koordination von Beziehungen der Punkte 2 und 3 durchaus - im Vergleich zur Zeit vor den Fusionen

$2 \quad$ Es gibt keine exakte Definition dessen, ab wann ein Programm als unterkritisch anzusehen ist, da dies je nach Kontext stark variiert. Ansatzpunkte sind jedoch die Laufzeit, die Anzahl der Förderfälle bezogen auf die Zielgruppe sowie das Budget für die Maßnahme. 
- als zufriedenstellend gewertet werden können, auch wenn die Abstimmung mit den Bundesländern nach wie vor als kompliziert angesehen wird, ist die Zielerreichung bei der Lösung von Koordinationsproblemen mit den Eigentümerministerien nur als bedingt erreicht einzustufen. Die noch verbleibenden Koordinationsprobleme beruhen auf einer unklaren Aufgabenteilung zwischen Agenturen und Ressorts, die zu einer Untersteuerung auf strategischer Ebene und einer teilweisen Übersteuerung auf operativer Ebene führt. Dies ist ein Befund, der bereits in der Systemevaluierung des österreichischen FTI-Systems im Jahr 2009 erhoben wurde (vgl. dazu die Spezialausgabe "Systemevaluierung" des fteval Journal for Research and Technology Policy Evaluation, Heft 34, 02.10). In der Zwischenzeit wurden zwar von der Politik bedeutende Anstrengungen zur Koordination und Steuerung der FTI-Politik insgesamt vorgenommen (ressortübergreifende FTI-Strategien, höhere Budgets etc.), auf Agenturebene wurden die gesetzlichen Grundlagen reformiert (KMUFörderungs- und Garantiegesetz, FTFG, FTI und FFG Richtlinien) sowie die Rahmenverträge überarbeitet, es erfolgten jedoch kaum Änderungen hinsichtlich der Governance der beiden Agenturen. Letzteres spricht einerseits die Tatsache an, dass die Agenden/Eigentümerinteressen der FFG sowie der aws nach wie vor auf zwei Ministerien verteilt sind und somit eigentlich einer intensive(re)n Koordination bedürfen, und andererseits eine strikt(er)e Trennung zwischen den strategischen sowie ausführenden Rollen erforderlich wäre, wobei sich die Ressorts auf die strategische Funktion konzentrieren und den Agenturen die Implementation überlassen sollten.

Die Erhöhung der politischen Steuerungsfähigkeit gegenüber den Zielgruppen ist im Vergleich zur Situation vor der Strukturreform als positiv zu bewerten. Durch die Schaffung der beiden Agenturen hat sich die Förderlandschaft vereinfacht, und es lassen sich einzelne Zielgruppen leichter in einer abgestimmten Art und Weise erreichen. Dies zeigt sich beispielsweise am Thema FuE-intensive Start-up Förderung. Das derzeit angebotene Portfolio wäre vor der Strukturreform auf vier bis fünf Organisationen aufgeteilt, mit allen einhergehenden Such- und Transaktionskosten sowie Komplikationen in der Administration für die Zielgruppe. Ein weiteres Beispiel stellt die Kombination von ERP-Krediten mit unterschiedlichen Garantieleistungen dar, die von den Unternehmen auch stark nachgefragt werden. Eine weitere Herausforderung stellt das missionsorientierte Förderportfolio bei sehr neuen bzw. dynamischen Themenfeldern dar, das teilweise einer besonderen Behandlung bedarf (z.B. in den Bereichen Energie und Mobilität), da die Entwicklung von capabilities/Kompetenzen, das community building sowie der Wissenstransfer bei missionsorientierten Programmen eine noch bedeutendere Rolle einnimmt und damit noch aktiver von den Agenturen unterstützt werden sollte.

Die Verbesserung der Umsetzungschancen von Regierungszielen, nämlich Effizienz und Intensivierung des Ressourceneinsatzes (Effektivität), stellt die Funktionalität der Umsetzungsebene in den Mittelpunkt der Betrachtung. Hier ist festzustellen, dass die beiden Agenturen, gegeben den ihnen gesetzten Rahmenbedingungen, durchaus erfolgreich agiert haben. Bezüglich der Effizienz des Handelns wurde die Abwicklung der Förder- und Finanzierungsportfolios über die Jahre deutlich modernisiert und die Zielgruppen sind mit der Kommunikation und Dienstleistung der Agenturen grosso modo sehr zufrieden. Die Abwicklungskosten haben sich bei der FFG im Beobachtungszeitraum (2006-2016) in Relation zu den Fördermitteln kontinuierlich reduziert, trotz gestiegener Aufgabenvielfalt. Für die aws lässt sich festhalten, dass die Implementierung einer Vielzahl moderner Management-Instrumente zu insgesamt optimierten und damit effizienteren Prozessen geführt hat. Dies zeigt sich nicht nur an einer Halbierung des time-to-contract, sondern auch anhand von deutlichen Produktivitätssteigerungen.

Aus methodisch-konzeptioneller Sicht lässt sich festhalten, dass sich die Orientierung an einer theoriebasierten Evaluation angesichts der Komplexität des Evaluierungsgegenstandes einschließlich der fehlenden Möglichkeit, eine kontrafaktische Situation herzustellen, bewährt hat. Die vielseitige empirische Strategie ist durch etablierte konzeptionelle Zugänge aus unterschiedlichen Literaturkreisen abzusichern, um den Erklärungsgehalt der Evaluierung zu erhöhen. Ebenfalls festhalten können wir, dass sich Unternehmens- und Organisationskulturen am besten durch ein verstehendes Vorgehen erfassen lassen, das auf qualitative Methoden wie beispielsweise narrative Interviews setzt. Wichtig ist auch, die einzelnen Hierarchieebenen im Evaluationsdesign adäquat abzubilden, was im hier vorliegenden Fall aus Zeit- und Kostengründen jedoch vor allem über Online-Surveys erfolgte. Dokumenten- und Datenanalysen sind zur Erfassung von Formalstrukturen, Leitbildern, Außendarstellungen unbedingt erforderlich, haben jedoch eine eher ergänzende Funktion. Abschließend halten wir es für zentral, die Innensicht durch die Außensicht, d.h. die Perspektive von nationalen und internationalen Stakeholdern sowie der Kundlnnen methodisch zu ergänzen.

Schlussendlich sei noch einmal darauf hingewiesen, dass die Evaluierung empfiehlt gemäß dem Leitsatz „Leistungsfähigkeit durch Eigenverantwortung" vor allem die finanzielle und operationale Autonomie der Agenturen zu stärken. Dies bedeutet einen Übergang von der Detailsteuerung hin zu einer ergebnisorientierten Steuerung "auf Abstand”, die mittels eines Globalbudgets zwar die gewünschten Wirkungen der Agentur mittels Zielformulierung und der Definition von Wirkungsindikatoren vorgibt, die Verteilung des Budgets auf Instrumente jedoch großteils der Agentur überlässt. Der zentrale Vorteil dieser Zukunftsoption läge darin, dass eine höhere Flexibilität der Umsetzung in der Agentur zu klarer kommunizierbaren Produkten, eine Beschleunigung von Prozessen zu einer besseren Zielgruppenansprache, und kurze Lernschleifen sowie eine flexible Kombination von Instrumenten zu einer größeren Hebelwirkung der eingesetzten Fördermittel führen können. In diesem Zusammenhang ist auch festzuhalten, dass dieses Modell bereits stark verbreitet ist, und insbesondere in den skandinavischen Ländern, sowie nun auch in der Schweiz, umgesetzt wird.

\section{REFERENZEN}

Braun, D.; Gilardi, F. (2002): Delegation aus der Sicht der PrinzipalAgent Theorie, in: Politische Vierteljahresschrift, 43 (1), 147-161.

Braun, D. (1993): Who Governs Intermediary Agencies? Principal-Agent Relations in Research Policy-Making, in: Journal of Public Policy, 13 (2), 135-162.

Braun, D. (1998): The Role of Funding Agencies in the Cognitive Development of Science, in: Research Policy, 27, 807-821.

fteval Journal for Research and Technology Policy Evaluation (2010). Spezialausgabe zur "Systemevaluierung, Heft 34, 02.10

Hasse, R.; Krücken, G. (2005): Neo-Institutionalismus. 2., überarbeitete Aufl., Bielefeld. 
Hochfeld, K.; Kaiser, S.; Schraudner, M. (2014) (Hrsg.): Unternehmenskulturen verändern - Vielfalt erreichen. Fraunhofer Verlag.

Koch, S. (2009): Die Bausteine neo-institutionalistischer Organisationstheorie - Begriffe und Konzepte im Lauf der Zeit. In: Koch, S.; Schemmmann, M. (Hrsg.): Neo-Institutionalismus in der Erziehungswissenschaft., S. 110-131. Springer Verlag.

Lane, J.-E. (2013): The Principal-Agent Approach to Politics: Policy Implementation and Public Policy-Making, in: Open Journal of Political Science, 3 (2), 85-89.

Leeuw, F. (2003), Reconstructing Program Theories: Methods Available and Problems to be Solved, in «American Journal of Evaluation», n. 24(1), pp. 5-20.

Leeuw, F. (2012): Linking theory-based evaluation and contribution analysis: Three problems and a few solution, Evaluation 18(3), p. 348-363.

Mayer, S.; Ruhland, S.; Fischl, I.; Sheikh, S. (2009): Governance in der FTI-Politik im Wechselspiel zwischen Ministerien und Agenturen. Wien

Meyer, J.W.; Rowan, B. (1977): Institutional organizations: formal structure as myth and ceremony. In: American Journal of Sociology, 83, 340-63.

Patton, M.0. (2002): Qualitative Research and Evaluation Methods, 2. Ausgabe. Newbury Park, CA: Sage.

Pichler, R. (2016): Agencification revisited. A Case for the Evaluation of FFG as an Agency. fteval Journal for Research and Technology Policy Evaluation, 41, 37-41

Pichler, R. (2014): Delegierte Koordination. Zur Koordinationsfunktion intermediärer Organisationen in der österreichischen Forschungsförderung, in: Österreichische Zeitschrift für Politikwissenschaft (ÖZP), 43 (4), 329-346.

Schein, E. (1985): Organizational Culture and Leadership. A Dynamic View, San Francisco etc.: Jossey-Bass.

Schein, E. (2003): Organisationskultur. The Ed Schein Corporate Culture Survival Guide, EHP Bergisch Gladbach.

Titscher, S.; Meyer, M.; Mayrhofer, W. (2008). Organisationsanalyse. Konzepte und Strategien. Wien: Facultas

Weiss, C.H. (1997a): How can theory-based evaluation make a greater headway? Evaluation Review Vol 21, 1997, p. 501-524

Weiss C.H. (1997b): Theory-based evaluation: Past, present and future. New Directions for Evaluation 76:41-55.

\section{AUTORiNNEN}

SUSANNE BÜHRER, STEPHANIE DAIMER, KNUT KOSCHATZKY

Fraunhofer ISI, Karlsruhe

E: Susanne.Buehrer@isi.fraunhofer.de

PETER KAUFMANN, SASCHA RUHLAND, SONJA SHEIKH

KMU Forschung Austria, Wien

E: p.kaufmann@kmuforschung.ac.at 